\title{
BOUNDEDNESS OF IMPULSIVE FUNCTIONAL DIFFERENTIAL EQUATIONS WITH VARIABLE IMPULSIVE PERTURBATIONS
}

\author{
I. M. STAMOVA
}

(Received 27 August 2007)

\begin{abstract}
In the present paper an initial value problem for impulsive functional differential equations with variable impulsive perturbations is considered. By means of piecewise continuous functions coupled with the Razumikhin technique, sufficient conditions for boundedness of solutions of such equations are found.
\end{abstract}

2000 Mathematics subject classification: 34K45.

Keywords and phrases: boundedness, impulsive functional differential equations, variable impulsive perturbations.

\section{Introduction}

Impulsive equations have many applications in physics, biology, medicine and other sciences. The theory of impulsive differential equations has been developed intensively; see, for instance, [3, 4, 11, 15].

The impulsive functional differential equations are a natural generalization of functional differential equations without impulses and of impulsive ordinary differential equations without delay. They are adequate mathematical models of various real processes and phenomena, characterized by the fact that their state changes by jumps and by the dependence of the process on its history at each moments of time. The qualitative theory of functional differential equations has developed very intensively. We refer to $[9,10,12,14]$ for the study of functional differential equations.

The theory of impulsive functional differential equations is also undergoing rapid development. See, for example, [1, 5-8, 13, 16-18] and the references cited therein. Many results on the stability of solutions of such equations have been obtained. Concerning the boundedness of impulsive functional differential equations, a few results have appeared for impulsive functional differential equations with fixed moments of impulse effect $[8,13]$. In the investigation of the impulsive functional differential equations with variable impulsive perturbations there arise a number

(C) 2008 Australian Mathematical Society 0004-9727/08 \$A2.00 + 0.00 
of difficulties related to the phenomena of 'beating' of the solutions, bifurcation, loss of the property of autonomy, and so on. The wider application, however, of equations of this type requires the formulation of effective criteria for boundedness of their solutions.

In the present paper the problem of boundedness of solutions of impulsive functional differential equations with variable impulsive perturbations is considered by means of Lyapunov's direct method. We use the picewise continuous Lyapunov functions. Moreover, the technique of investigation essentially depends on the choice of minimal subsets of a suitable space of piecewise continuous functions, by the elements of which the derivatives of Lyapunov's functions are estimated [8, 13, 18]. It is well known that the Lyapunov-Razumikhin function method has been widely used in the treatment of the stability of functional differential equations without impulses [9, 12, 14].

\section{Preliminary notes and definitions}

Let $R^{n}$ be the $n$-dimensional Euclidean space with norm $|\cdot|$ and $R_{+}=[0, \infty)$. Let $t_{0} \in R, r>0$. Consider the following system

$$
\begin{cases}\dot{x}(t)=f\left(t, x_{t}\right), & t \neq \tau_{k}(x(t)), \\ \Delta x(t)=I_{k}(x(t-0)), & t=\tau_{k}(x(t)), k=1,2, \ldots,\end{cases}
$$

where $f:\left(t_{0}, \infty\right) \times D \rightarrow R^{n}$;

$D=\left\{\phi:[-r, 0] \rightarrow R^{n}, \phi(t)\right.$ is continuous everywhere except at a finite

number of points $\tilde{t}$ at which $\phi(\tilde{t}-0)$ and $\phi(\widetilde{t}+0)$ exist and $\phi(\tilde{t}-0)=\phi(\widetilde{t})\}$;

$I_{k}: R^{n} \rightarrow R^{n}, k=1,2, \ldots ; \tau_{k}: R^{n} \rightarrow\left(t_{0}, \infty\right) ; \Delta x(t)=x(t+0)-x(t-0)$, and for $t>t_{0}, x_{t} \in D$ is defined by $x_{t}=x(t+s),-r \leq s \leq 0$.

Let $\tau_{0}(x) \equiv t_{0}$ for $x \in R^{n}$.

We introduce the following conditions:

(H1) $\tau_{k} \in C\left[R^{n},\left(t_{0}, \infty\right)\right], k=1,2, \ldots$;

(H2) $t_{0}<\tau_{1}(x)<\tau_{2}(x)<\cdots, x \in R^{n}$;

(H3) $\tau_{k}(x) \rightarrow \infty$ as $k \rightarrow \infty$ uniformly on $x \in R^{n}$.

Assuming that conditions (H1), (H2), and (H3) are fulfilled, we introduce the following notation:

$$
\begin{gathered}
G_{k}=\left\{(t, x) \in\left[t_{0}, \infty\right) \times R^{n}: \tau_{k-1}(x)<t<\tau_{k}(x)\right\}, \quad k=1,2, \ldots, \\
\sigma_{k}=\left\{(t, x) \in\left[t_{0}, \infty\right) \times R^{n} \mid t=\tau_{k}(x)\right\},
\end{gathered}
$$

that is, $\sigma_{k}, k=1,2, \ldots$, are hypersurfaces of the equations $t=\tau_{k}(x(t))$.

Let $\varphi_{0} \in D$. Denote by $x(t)=x\left(t ; t_{0}, \varphi_{0}\right)$ the solution of system (2.1) satisfying the initial conditions

$$
\left\{\begin{array}{l}
x\left(t ; t_{0}, \varphi_{0}\right)=\varphi_{0}\left(t-t_{0}\right), \quad t_{0}-r \leq t \leq t_{0}, \\
x\left(t_{0}+0 ; t_{0}, \varphi_{0}\right)=\varphi_{0}(0),
\end{array}\right.
$$


and by $J^{+}\left(t_{0}, \varphi_{0}\right)$ the maximal interval of the type $\left(t_{0}, \beta\right)$, at which the solution $x\left(t ; t_{0}, \varphi_{0}\right)$ is defined.

The precise description of the solution $x\left(t ; t_{0}, \varphi_{0}\right)$ of $(2.1),(2.2)$ is given in $[5,16]$.

We introduce the following notation:

$$
\begin{gathered}
C_{0}=C\left[\left[t_{0}-r, t_{0}\right], R^{n}\right], \\
\|\varphi\|=\max _{t \in\left[t_{0}-r, t_{0}\right]}\left|\varphi\left(t-t_{0}\right)\right| \text { is the norm of the function } \varphi \in C_{0}, \\
S_{\rho}=\left\{x \in R^{n}:|x|<\rho, \rho>0\right\}, \\
S_{\rho}^{c}=\left\{x \in R^{n}:|x| \geq \rho, \rho>0\right\}, \\
K=\left\{a \in C\left[R_{+}, R_{+}\right]: a(r) \text { is strictly increasing and } a(0)=0\right\} .
\end{gathered}
$$

We then introduce the following conditions:

(H4) $f \in C\left[\left(t_{0}, \infty\right) \times D, R^{n}\right]$;

(H5) the function $f$ is Lipschitz continuous with respect to its second argument in $\left(t_{0}, \infty\right) \times D$ uniformly on $t \in\left(t_{0}, \infty\right)$

(H6) $|f(t, \tilde{x})| \leq L<\infty$ for $(t, \tilde{x}) \in\left(t_{0}, \infty\right) \times D, L>0$;

(H7) for any $k=1,2, \ldots$ the following inequality is valid

$$
\left|I_{k}\left(x_{1}\right)-I_{k}\left(x_{2}\right)\right| \leq c\left|x_{1}-x_{2}\right|, \quad x_{1}, x_{2} \in R^{n}, c>0
$$

(H8) the integral curves of the system (2.1) meet successively each one of the hypersurfaces $\sigma_{1}, \sigma_{2}, \ldots$ exactly once.

Condition (H8) guarantees the absence of the phenomenon of 'beating' of the solutions to the system (2.1), that is, where a given integral curve meets one and the same hypersurface more than once or infinitely many times. Efficient sufficient conditions which guarantee the absence of 'beating' of the solutions of such systems are given in [2].

Let $t_{1}, t_{2}, \ldots\left(t_{0}<t_{1}<t_{2}<\cdots\right)$ be the moments in which the integral curve $\left(t, x\left(t ; t_{0}, \varphi_{0}\right)\right)$ of the problem $(2.1),(2.2)$ meets the hypersurfaces $\sigma_{k}, k=1,2, \ldots$

We shall note that if conditions (H1)-(H8) are met, then $t_{k} \rightarrow \infty$ as $k \rightarrow \infty$ and $J^{+}\left(t_{0}, \varphi_{0}\right)=\left(t_{0}, \infty\right)[2,6]$.

We shall use the following definitions of boundedness of the solutions of (2.1).

Definition 1. Let $x\left(t ; t_{0}, \varphi_{0}\right)$ be any solution of (2.1). The system (2.1) is said to be: 
(a) uniformly bounded if

$$
\begin{gathered}
(\forall \alpha>0)(\exists \beta=\beta(\alpha)>0)\left(\forall t_{0} \in R_{+}\right) \\
\left(\forall \varphi_{0} \in C_{0}:\left\|\varphi_{0}\right\|<\alpha\right):\left|x\left(t ; t_{0}, \varphi_{0}\right)\right|<\beta, \quad t \geq t_{0} ;
\end{gathered}
$$

(b) quasi-uniformly ultimately bounded if

$$
\begin{gathered}
(\exists B>0)(\forall \alpha>0)(\exists T=T(\alpha)>0)\left(\forall t_{0} \in R_{+}\right) \\
\left(\forall \varphi_{0} \in C_{0}:\left\|\varphi_{0}\right\|<\alpha\right):\left|x\left(t ; t_{0}, \varphi_{0}\right)\right|<B, \quad t \geq t_{0}+T ;
\end{gathered}
$$

(c) uniformly ultimately bounded if (a) and (b) hold together.

Let $I=\left[t_{0}-r, \infty\right)$ and $I_{0}=\left[t_{0}, \infty\right)$. In what follows we shall use the class $V_{0}$ of piecewise continuous auxiliary functions $V: I_{0} \times R^{n} \rightarrow R_{+}$which are analogues of Lyapunov's functions.

Definition 2. We say that the function $V:\left[I_{0}, \infty\right) \times R^{n} \rightarrow R_{+}$, belongs to the class $V_{0}$ if the following conditions are fulfilled.

(1) The function $V$ is continuous in

$$
\bigcup_{k=1}^{\infty} G_{k}, \quad V(t, 0)=0, t \in\left[I_{0}, \infty\right),
$$

and locally Lipschitz continuous with respect to its second argument $x$ on each of the sets $G_{k}, k=1,2, \ldots$

(2) For each $k=1,2, \ldots$ and $\left(t_{0}^{*}, x_{0}^{*}\right) \in \sigma_{k}$ there exist the finite limits

$$
V\left(t_{0}^{*}-0, x_{0}^{*}\right)=\lim _{\substack{(t, x) \rightarrow\left(t_{0}^{*}, x_{0}^{*}\right) \\(t, x) \in G_{k}}} V(t, x), \quad V\left(t_{0}^{*}+0, x_{0}^{*}\right)=\lim _{\substack{(t, x) \rightarrow\left(t_{0}^{*}, x_{0}^{*}\right) \\(t, x) \in G_{k+1}}} V(t, x) .
$$

(3) For each $k=1,2, \ldots$ the following equalities are valid:

$$
V\left(t_{0}^{*}-0, x_{0}^{*}\right)=V\left(t_{0}^{*}, x_{0}^{*}\right) .
$$

Let $J \subset R$ be an interval. Define the following classes of functions:

$$
\begin{aligned}
P C\left[J, R^{n}\right]= & \left\{\sigma \mid J \rightarrow R^{n}: \sigma(t)\right. \text { is continuous everywhere except at } \\
& \text { some points } t_{k} \text { where } \sigma\left(t_{k}-0\right) \text { and } \sigma\left(t_{k}+0\right) \text { exist and } \\
& \left.\sigma\left(t_{k}-0\right)=\sigma\left(t_{k}\right), k=1,2, \ldots\right\} ; \\
\Omega_{P}= & \left\{x \in P C\left[I_{0}, R^{n}\right] \mid V(s, x(s)) \leq P(V(t, x(t))),\right. \\
& \left.t-r \leq s \leq t, t \in I_{0}, V \in V_{0}\right\},
\end{aligned}
$$

where $P(u)$ is continuous on $R_{+}$, nondecreasing in $u$, and $P(u)>u$ for $u>0$.

Let $V \in V_{0}$. For $x \in P C\left[I_{0}, R^{n}\right]$ and $t \in I_{0}, t \neq t_{k}(x(t)), k=1,2, \ldots$, we define the function

$$
D_{-} V(t, x(t))=\lim _{h \rightarrow 0^{-}} \inf h^{-1}\left[V\left(t+h, x(t)+h f\left(t, x_{t}\right)\right)-V(t, x(t))\right] .
$$




\section{Main results}

THEOREM 3.1. Assume that the following conditions hold.

(1) Assumptions (H1)-(H8) are valid.

(2) For $\rho>0$, there exists $V \in V_{0}$ such that

$$
a(|x|) \leq V(t, x) \leq b(|x|), \quad(t, x) \in I_{0} \times S_{\rho}^{c},
$$

where $a, b \in K$ and $a(r) \rightarrow \infty$ as $t \rightarrow \infty$.

(3) For $t \geq t_{0},(t, x(t)) \in I_{0} \times S_{\rho}^{c}$ and $x \in \Omega_{P}$,

$$
D_{-} V(t, x(t)) \leq-c(|x(t)|), \quad t \neq \tau_{k}(x(t)), k=1,2 \ldots,
$$

where $c \in K$.

(4) For $t \geq t_{0},(t, x(t)) \in I_{0} \times S_{\rho}^{c}$,

$$
V\left(t+0, x(t)+I_{k}(x(t))\right) \leq V(t, x(t)), \quad t=\tau_{k}(x(t)), k=1,2 \ldots
$$

Then the system (2.1) is uniformly bounded.

ProOF. Let $\alpha \geq \rho$ be given. Choose $\beta=\beta(\alpha)>0$ so that

$$
\beta>\max \left\{\alpha, a^{-1}(b(\alpha))\right\} .
$$

Let $t_{0} \in R_{+}$and $\varphi_{0} \in C_{0}$. Consider the solution $x(t)=x\left(t ; t_{0}, \varphi_{0}\right)$ of (2.1) with $\left\|\varphi_{0}\right\|<\alpha$. Clearly,

$$
\left|x\left(t_{0}+0 ; t_{0}, \varphi_{0}\right)\right|=\left|\varphi_{0}(0)\right| \leq\left\|\varphi_{0}\right\|<\alpha<\beta .
$$

We claim that

$$
|x(t)|<\beta, \quad t \geq t_{0} .
$$

If this is not true, then there exists some solution $x(t)=x\left(t ; t_{0}, \varphi_{0}\right)$ of (2.1) with $\left\|\varphi_{0}\right\|<\alpha$ and a $t^{*}>t_{0}$ such that $\left|x\left(t^{*} ; t_{0}, \varphi_{0}\right)\right| \geq \beta$. Thus there exist

$$
s_{1}, s_{2}, t_{0} \leq s_{1}<s_{2} \leq t^{*}
$$

such that

$$
\left|x\left(s_{1}+0\right)\right| \geq \alpha, \quad\left|x\left(s_{1}\right)\right| \leq \alpha, \quad\left|x\left(s_{2}+0\right)\right| \geq \beta,
$$

and

$$
x(t) \in S_{\beta} \cap S_{\alpha}^{c}, \quad t \in\left[s_{1}, s_{2}\right) .
$$

First, we show that

$$
V\left(s_{1}+0, x\left(s_{1}+0\right)\right)<a(\beta) .
$$


If $s_{1} \neq t_{k}$, then $\left|x\left(s_{1}\right)\right|=\alpha$, and, by (3.1),

$$
V\left(s_{1}, x\left(s_{1}\right)\right) \leq b\left(\left|x\left(s_{1}\right)\right|\right)=b(\alpha)<a(\beta) .
$$

If $s_{1}=t_{k}$ for some $k$, then $\left|x\left(s_{1}\right)\right| \leq \alpha$ and

$$
V\left(s_{1}, x\left(s_{1}\right)\right) \leq b\left(\left|x\left(s_{1}\right)\right|\right) \leq b(\alpha)<a(\beta) .
$$

Thus by (3.3) we obtain

$$
V\left(s_{1}+0, x\left(s_{1}+0\right)\right)<a(\beta) .
$$

Next, we wish to show that

$$
V(t+0, x(t+0))<a(\beta), \quad t \in\left[s_{1}, s_{2}\right] .
$$

Suppose that this is not true and let

$$
\mu=\inf \left\{s_{2} \geq t>s_{1} \mid V(t+0, x(t+0)) \geq a(\beta)\right\} .
$$

We discuss two possibilities:

(A) $\mu \neq t_{k}, k=1,2, \ldots$ Since $V(t, x(t))$ is continuous at $\mu$,

$$
V(\mu+0, x(\mu+0))=a(\beta) .
$$

Thus for $h<0$ with $|h|$ small enough the inequality

$$
V(\mu+h, x(\mu+h))<a(\beta)
$$

holds which implies that

$$
D_{-} V(\mu, x(\mu))=\lim _{h \rightarrow 0^{-}} \inf h^{-1}[V(\mu+h, x(\mu+h))-V(\mu, x(\mu))] \geq 0 .
$$

It is clear from the choice of $\mu$ that

$$
P(V(\mu, x(\mu)))>V(\mu, x(\mu)) \geq V(s, x(s)), \quad s_{1} \leq s \leq \mu .
$$

Thus, we obtain, using (3.2) and (3.4),

$$
D_{-} V(\mu, x(\mu)) \leq-c(|x(\mu)|) \leq-c(\alpha)<0,
$$

which contradicts (3.7).

(B) $\mu=t_{k}$ for some $k=1,2, \ldots$ We must have

$$
V\left(t_{k}+0, x\left(t_{k}+0\right)\right)=a(\beta) .
$$

In fact, if $V\left(t_{k}+0, x\left(t_{k}+0\right)\right)>a(\beta)$, then, by assumption (3.3),

$$
V\left(t_{k}, x\left(t_{k}\right)\right)>a(\beta) .
$$


Since $V(t, x(t))$ is left continuous at $t_{k}$, it follows that there exists $\tilde{\mu}<t_{k}$ such that $V(\tilde{\mu}+0, x(\tilde{\mu}+0)) \geq a(\beta)$ which contradicts the choice of $\mu$.

Now for $h<0$ with $|h|$ small enough so that $t_{k}+h \in\left(t_{k-1}, t_{k}\right)$

$$
V\left(t_{k}+h, x\left(t_{k}+h\right)\right)<a(\beta) .
$$

From (3.3),

$$
V(\mu, x(\mu))=V\left(t_{k}, x\left(t_{k}\right)\right) \geq V\left(t_{k}+0, x\left(t_{k}+0\right)\right) .
$$

Hence

$$
\begin{aligned}
& V(\mu+h, x(\mu+h))-V(\mu, x(\mu)) \\
& \quad \leq V\left(t_{k}+h, x\left(t_{k}+h\right)\right)-V\left(t_{k}+0, x\left(t_{k}+0\right)\right)<0,
\end{aligned}
$$

which implies that

$$
\begin{aligned}
D_{-} V(\mu, x(\mu)) & =\lim _{h \rightarrow 0^{-}} \inf h^{-1}[V(\mu+h, x(\mu+h))-V(\mu, x(\mu))] \\
& \geq \lim _{h \rightarrow 0^{-}} \inf h^{-1}\left[V\left(t_{k}+h, x\left(t_{k}+h\right)\right)-V\left(t_{k}+0, x\left(t_{k}+0\right)\right)\right] \geq 0 .
\end{aligned}
$$

Since

$$
P(V(\mu, x(\mu)))>V(\mu, x(\mu)) \geq V(s, x(s)), \quad s_{1} \leq s \leq \mu,
$$

we obtain, using (3.2),

$$
D_{-} V(\mu, x(\mu)) \leq-c(|x(\mu)|) \leq-c(\alpha)<0,
$$

which contradicts (3.8). Therefore (3.6) holds.

On the other hand, using (3.1), we obtain

$$
V\left(s_{2}+0, x\left(s_{2}+0\right)\right) \geq a\left(\left|x\left(s_{2}+0\right)\right|\right) \geq a(\beta),
$$

which contradicts (3.6). Thus

$$
|x(t)|<\beta, \quad t \geq t_{0},
$$

for any solution $x(t)=x\left(t ; t_{0}, \varphi_{0}\right)$ of (2.1) with $\left\|\varphi_{0}\right\|<\alpha$ and the system (2.1) is uniformly bounded. This completes the proof of Theorem 3.1.

THEOREM 3.2. Assume that the following conditions hold.

(1) Conditions (1), (2) and (4) of Theorem 3.1 are valid.

(2) For $t \geq t_{0},(t, x(t)) \in I_{0} \times S_{\rho}^{c}$ and $x \in \Omega_{P}$,

$$
D_{-} V(t, x(t)) \leq M-c(|x(t)|), \quad t \neq \tau_{k}(x(t)), k=1,2, \ldots,
$$

where $c \in K$ and $M=$ constant.

Then the system (2.1) is uniformly ultimately bounded. 
PROOF. We begin by proving the uniform boundedness of the system (2.1).

Let $\rho>0$ be sufficiently large that

$$
M-c(\rho)<0
$$

Let $\alpha>\max \left\{\rho, c^{-1}(M)\right\}$ be given. Choose $\beta=\max \left\{\alpha, a^{-1}(b(\alpha))\right\}$ for any $t_{0} \in R_{+}$ and $\varphi_{0} \in C_{0},\left\|\varphi_{0}\right\|<\alpha$. Clearly, $\left\|\varphi_{0}\right\|<\alpha \leq \beta$.

Now suppose that there exist a solution $x(t)=x\left(t ; t_{0}, \varphi_{0}\right)$ of $(2.1)$ and a $t^{*}>t_{0}$ such that $\left|x\left(t^{*} ; t_{0}, \varphi_{0}\right)\right| \geq \beta$. Then there exist $s_{1}, s_{2}, t_{0} \leq s_{1}<s_{2} \leq t^{*}$ such that

$$
\left|x\left(s_{1}+0\right)\right| \geq \alpha, \quad\left|x\left(s_{1}\right)\right| \leq \alpha, \quad\left|x\left(s_{2}+0\right)\right| \geq \beta,
$$

and

$$
x(t) \in S_{\beta} \cap S_{\alpha}^{c}, \quad t \in\left[s_{1}, s_{2}\right) .
$$

First, we can prove by same arguments as in the proof of Theorem 3.1 that

$$
V\left(s_{1}+0, x\left(s_{1}+0\right)\right)<a(\beta) .
$$

Next, we show that

$$
V(t+0, x(t+0))<a(\beta), \quad t \in\left[s_{1}, s_{2}\right] .
$$

Suppose that this is not true and let

$$
\sigma=\inf \left\{s_{2} \geq t>s_{1} \mid V(t+0, x(t+0)) \geq a(\beta)\right\} .
$$

We consider two cases.

(A) $\sigma \neq t_{k}, k=1,2, \ldots$ We can see that

$$
V(\sigma+0, x(\sigma+0))=a(\beta) \quad \text { and } \quad D_{-} V(\sigma, x(\sigma)) \geq 0 .
$$

Then there exists an $s_{1}^{*} \in\left(s_{1}, s_{2}\right]$ such that

$$
V(s, x(s)) \leq P(V(\sigma, x(\sigma))), \quad s_{1}^{*} \leq s \leq \sigma .
$$

Since $|x(\sigma)| \geq \alpha$, we obtain, by condition (2) of Theorem 3.2,

$$
\begin{aligned}
& D_{-} V(\sigma, x(\sigma)) \leq M-c(|x(\sigma)|) \leq M-c(\alpha) \\
& \quad<M-c\left(\max \left\{\rho, c^{-1}(M)\right\}=M-\max \{M, c(\rho)\} \leq 0,\right.
\end{aligned}
$$

which contradicts (3.9).

(B) $\sigma=t_{j}$ for some $j \in\{1,2, \ldots, k, \ldots\}$. We can obtain a contradiction by the analogous arguments as in the proof of Theorem 3.1. 
On the other hand, using (3.1) we obtain

$$
V\left(s_{2}+0, x\left(s_{2}+0\right)\right) \geq a\left(\left|x\left(s_{2}+0\right)\right|\right) \geq a(\beta),
$$

which contradicts the fact that $V(t+0, x(t+0))<a(\beta), t \in\left[s_{1}, s_{2}\right]$. Thus

$$
|x(t)|<\beta, \quad t \geq t_{0},
$$

for any solution $x(t)=x\left(t ; t_{0}, \varphi_{0}\right)$ of (2.1) with $\left\|\varphi_{0}\right\|<\alpha$ and the system (2.1) is uniformly bounded.

The uniform boundedness of the system (2.1) means that there exists a positive number $B$ such that for each $t_{0} \in R_{+}$,

$$
\left\|\varphi_{0}\right\|<\rho \text { implies }\left|x\left(t ; t_{0}, \varphi_{0}\right)\right|<B, \quad t \geq t_{0} .
$$

Now we consider the solution $x(t)=x\left(t ; t_{0}, \varphi_{0}\right)$ of (2.1) with $\left\|\varphi_{0}\right\|<\alpha$, where $\alpha$ is an arbitrary number and $\alpha>\rho$. Then there exists a positive number

$$
\beta=\beta(\alpha)>\max \left\{B, a^{-1}(b(\alpha))\right\}
$$

such that

$$
|x(t)|<\beta, \quad t \geq t_{0} .
$$

Let the function $P: R_{+} \rightarrow R_{+}$be a continuous and nondecreasing on $R_{+}$, and $P(u)>u$ as $u>0$. We set

$$
\lambda=\inf \{P(u)-u \mid a(B) \leq u \leq a(\beta)\} .
$$

Then

$$
P(u)>u+\lambda \quad \text { as } a(B) \leq u \leq a(\beta),
$$

and we choose the integer $N$ such that

$$
a(B)+(N-1) \lambda>a(\beta) .
$$

Choose $T=N(\lambda / c(\rho))$ and define

$$
t_{m}=t_{0}+m \frac{\lambda}{c(\rho)-M}, \quad m=0,1,2, \ldots, N
$$

then $t_{N}=t_{0}+T$. We show that

$$
\left|x\left(t ; t_{0}, \varphi_{0}\right)\right|<B, \quad t \geq t_{0}+T .
$$

Suppose that is not true, then there exists a $t^{*}>t_{0}+T$ such that

$$
\left|x\left(t^{*} ; t_{0}, \varphi_{0}\right)\right| \geq B
$$


Then in view of (3.10),

$$
\left|x\left(t ; t_{0}, \varphi_{0}\right)\right| \geq \rho, \quad t \in\left[t_{0}, t^{*}\right] .
$$

By (3.1) we obtain

$$
V\left(t_{0}+0, \varphi_{0}(0)\right) \leq b\left(\left|\varphi_{0}(0)\right|\right) \leq b\left(\left\|\varphi_{0}\right\|\right)<b(\alpha)<a(\beta) .
$$

We claim that

$$
V(t, x(t))<a(\beta), \quad t \in\left[t_{0}, t^{*}\right] .
$$

Suppose that this is not true and let

$$
\xi=\inf \left\{t^{*} \geq t \geq t_{0} \mid V(t, x(t)) \geq a(\beta)\right\} .
$$

If $\xi \neq t_{k}, k=1,2, \ldots$, then by the definition of $\xi$,

$$
V(\xi, x(\xi))=a(\beta) .
$$

Thus for $h<0$ with $|h|$ small enough the inequality

$$
V(\xi+h, x(\xi+h))<a(\beta)
$$

holds and consequently

$$
D_{-} V(\xi, x(\xi))=\lim _{h \rightarrow 0^{-}} \inf h^{-1}[V(\xi+h, x(\xi+h))-V(\xi, x(\xi))] \geq 0 .
$$

It is clear from the choice of $\xi$ that

$$
P(V(\xi, x(\xi)))>V(\xi, x(\xi)) \geq V(s, x(s)), \quad t_{0} \leq s \leq \xi .
$$

By (3.14) we see that $x(\xi) \in S_{\rho}^{c}$. Thus by condition (2) of Theorem 3.2

$$
D_{-} V(\xi, x(\xi)) \leq M-c(|x(\xi)|) \leq M-c(\rho)<0,
$$

which contradicts (3.16).

If $\xi=t_{j}$ for some $j \in\{1,2, \ldots, k, \ldots\}$, then the same arguments as in the proof of Theorem 3.1 show that

$$
V\left(t_{k}+0, x\left(t_{k}+0\right)\right)=a(\beta)
$$

and

$$
D_{-} V(\xi, x(\xi)) \geq \lim _{h \rightarrow 0^{-}} \inf h^{-1}\left[V\left(t_{k}+h, x\left(t_{k}+h\right)\right)-V\left(t_{k}+0, x\left(t_{k}+0\right)\right)\right] \geq 0 .
$$


However

$$
P(V(\xi, x(\xi)))>V(\xi, x(\xi)) \geq V(s, x(s)), \quad t_{0} \leq s \leq \xi,
$$

and $x(\xi) \in S_{\rho}^{c}$. Thus by condition (2) of Theorem 3.2

$$
D_{-} V(\xi, x(\xi)) \leq M-c(|x(\xi)|) \leq M-c(\rho)<0,
$$

which contradicts (3.17). Hence (3.15) holds.

We next show that

$$
V(t, x(t))<a(\beta)+(N-m-1) \lambda, \quad t \in\left[t_{m}, t^{*}\right], m=0,1,2, \ldots, N-1 .
$$

From (3.12) and (3.15),

$$
V(t, x(t))<a(\beta)+(N-1) \lambda, \quad t \in\left[t_{0}, t^{*}\right],
$$

which shows the validity of (3.18) for $m=0$. We prove inequality (3.18) by induction. Suppose that for some integer $m, 0 \leq m<N-1$,

$$
V(t, x(t))<a(B)+(N-m-1) \lambda, \quad t \in\left[t_{m}, t^{*}\right] .
$$

First, we have to show that there exists $\tilde{t} \in\left[t_{m}, t_{m+1}\right]$ such that

$$
V(\tilde{t}, x(\widetilde{t}))<a(B)+(N-m-2) \lambda .
$$

If this is not true, then

$$
V(t, x(t)) \geq a(B)+(N-m-2) \lambda, \quad t \in\left[t_{m}, t_{m+1}\right] .
$$

By (3.15) and (3.21),

$$
a(B) \leq V(t, x(t)) \leq a(\beta), \quad t \in\left[t_{m}, t_{m+1}\right] .
$$

We consider two cases.

(A) $t \neq t_{k}, k=1,2, \ldots$, for all $t \in\left[t_{m}, t_{m+1}\right]$. Using (3.11), (3.19) and (3.21), we obtain

$$
\begin{aligned}
P(V(t, x(t))) & \geq V(t, x(t))+\lambda \geq a(B)+(N-m-1) \lambda \\
& >V(s, x(s)), \quad t_{m} \leq s \leq t, t \in\left[t_{m}, t_{m+1}\right] .
\end{aligned}
$$

By (3.14), we see that $x(t) \in S_{\rho}^{c}$ for $t \in\left[t_{m}, t_{m+1}\right] \subset\left[t_{0}, t^{*}\right]$. Thus it follows from condition (2) of Theorem 3.2 that

$$
\begin{aligned}
V\left(t_{m+1}, x\left(t_{m+1}\right)\right) & \leq V\left(t_{m}, x\left(t_{m}\right)\right)-\int_{t_{m}}^{t_{m+1}}[c(|x(s)|)-M] d s \\
& <a(B)+(N-m-1) \lambda-(c(\rho)-M)\left[t_{m+1}-t_{m}\right] \\
& \leq V\left(t_{m+1}, x\left(t_{m+1}\right)\right),
\end{aligned}
$$

which is a contradiction. 
(B) There exist $t_{k_{i}} \in\left[t_{m}, t_{m+1}\right]$ for $k_{i} \in\{1,2, \ldots, k, \ldots\}, i=1,2, \ldots, j ; j \geq 1$. By (3.11), (3.19) and (3.21),

$$
\begin{aligned}
P(V(t, x(t))) & \geq V(t, x(t))+\lambda \geq a(B)+(N-m-1) \lambda \\
& >V(s, x(s)), \quad t_{m} \leq s \leq t, t \in\left[t_{m}, t_{m+1}\right] .
\end{aligned}
$$

Since $x(t) \in S_{\rho}^{c}$ for $t \in\left[t_{m}, t_{m+1}\right]$, we have by condition (2) of Theorem 3.2 that

$$
\begin{aligned}
\int_{t_{m}}^{t_{m+1}} D_{-} V(s, x(s)) d s & \leq-\int_{t_{m}}^{t_{m+1}}[c(|x(s)|)-M] d s \\
& \leq-(c(\rho)-M)\left[t_{m+1}-t_{m}\right]=-\lambda .
\end{aligned}
$$

By (3.3) the inequalities

$$
V\left(t_{k_{i}}, x\left(t_{k_{i}}\right)\right)-V\left(t_{k_{i}}+0, x\left(t_{k_{i}}+0\right)\right) \geq 0, \quad i=1,2, \ldots, j,
$$

hold, giving

$$
\begin{aligned}
\int_{t_{m}}^{t_{m+1}} & D_{-} V(s, x(s)) d s \\
= & \int_{t_{k_{j}}}^{t_{m+1}} D_{-} V(s, x(s)) d s \\
& +\int_{t_{k_{j}-1}}^{t_{k_{j}}} D_{-} V(s, x(s)) d s \\
& +\cdots+\int_{t_{k_{1}}}^{t_{k_{2}}} D_{-} V(s, x(s)) d s+\int_{t_{m}}^{t_{k_{2}}} D_{-} V(s, x(s)) d s \\
\geq & V\left(t_{m+1}, x\left(t_{m+1}\right)\right)+\left[V\left(t_{k_{j}}, x\left(t_{k_{j}}\right)\right)-V\left(t_{k_{j}}+0, x\left(t_{k_{j}}+0\right)\right)\right] \\
& +\cdots+\left[V\left(t_{k_{1}}, x\left(t_{k_{1}}\right)\right)-V\left(t_{k_{1}}+0, x\left(t_{k_{1}}+0\right)\right)\right]-V\left(t_{m}, x\left(t_{m}\right)\right) \\
\geq & V\left(t_{m+1}, x\left(t_{m+1}\right)\right)-V\left(t_{m}, x\left(t_{m}\right)\right) .
\end{aligned}
$$

In view of (3.19), (3.21), (3.24) and (3.25), it follows that

$$
\begin{aligned}
V\left(t_{m+1}, x\left(t_{m+1}\right)\right) & \leq V\left(t_{m}, x\left(t_{m}\right)\right)-\lambda<a(B)+(N-m-1) \lambda-\lambda \\
& \leq V\left(t_{m+1}, x\left(t_{m+1}\right)\right) .
\end{aligned}
$$

The contradiction obtained shows that there exists a $\tilde{t} \in\left[t_{m}, t_{m+1}\right]$ such that (3.20) holds.

Next, we shall show that (3.20) implies that

$$
V(t, x(t))<a(B)+(N-m-2) \lambda, \quad \tilde{t} \leq t \leq t^{*}, \tilde{t} \in\left[t_{m}, t_{m+1}\right] .
$$

Suppose that this is not true and let

$$
\eta=\inf \left\{t^{*} \geq t \geq \tilde{t} \mid V(t, x(t)) \geq a(B)+(N-m-2) \lambda\right\} .
$$


Then we can obtain a contradiction by the same arguments as in the proof of the inequality (3.15). Hence we have proved

$$
V(t, x(t))<a(B)+(N-m-2) \lambda, \quad t \in\left[t_{m+1}, t^{*}\right] .
$$

By induction we see that (3.18) is true for any $m=0,1,2, \ldots, N-1$. Therefore we obtain

$$
V(t, x(t))<a(B), \quad t \in\left[t_{N-1}, t^{*}\right] .
$$

On the other hand, using (3.1),

$$
V\left(t^{*}, x\left(t^{*}\right)\right) \geq a\left(\left|x\left(t^{*}\right)\right|\right) \geq a(B),
$$

which contradicts (3.26). Therefore the system (2.1) is a uniformly ultimately bounded system. The proof is completed.

\section{An example}

Let $t_{0} \geq 0$ and $\tau_{0}(x) \equiv t_{0}$ for $x \in R$. Assume that the functions $\tau_{k}$ satisfy the conditions (H1), (H2), (H3) and (H8). Consider

$$
\tau_{k}(x)=2-2^{-k}-\frac{1}{1+x^{2}}, \quad x \in R, k=1,2, \ldots
$$

It is easy to check that conditions (H1), (H2), and (H3) are fulfilled.

Consider the nonlinear system

$$
\begin{cases}\dot{x}(t)=-3 \xi x^{4}(t)+\int_{t_{0}}^{t} p(t, s) x^{3}(s) d s+q(t), & t \neq \tau_{k}(x(t)), \\ \Delta x(t)=-\beta_{k} x(t-0), & t=\tau_{k}(x(t)), k=1,2, \ldots,\end{cases}
$$

where $\xi>0 ; 0 \leq \beta_{k} \leq 2, k=1,2, \ldots ; q \in C\left[R_{+}, R\right],|q(t)| \leq M$ for some constant $M>0 ; p \in C\left[R_{+} \times R_{+}, R_{+}\right]$.

Note that for the given choice of the functions $\tau_{k}$, the integral curves of the system (4.1) meet successively each one of the curves $\sigma_{1}, \sigma_{2}, \ldots$ exactly once [2].

Assume that there exists a constant $\mu>1$ such that

$$
\int_{t_{0}}^{t} p(t, s) d s \leq \frac{\xi}{\mu^{3}} .
$$

Then (4.2) is a sufficient condition for uniform ultimate boundedness of the system (4.1). In fact, we can shoose $a(r)=b(r)=r, c(r)=2 \xi r^{4}$. Let $V(t, x)=|x|$, $S_{\rho}^{c}=\{x \in R:|x|>1\}$ and denote

$$
P(u)=\mu \cdot u
$$


Thus, using (4.2),

$$
\begin{aligned}
V_{(4.1)}^{\prime}(t, x(t)) & \leq-3 \xi\left|x^{4}(t)\right|+\int_{t_{0}}^{t} p(t, s)\left|x^{3}(s)\right| d s+|q(t)| \\
& \leq-3 \xi|x(t)|^{4}+\mu^{3}|x(t)|^{3} \int_{t_{0}}^{t} p(t, s) d s+|q(t)| \\
& \leq-3 \xi|x(t)|^{4}+\mu^{3}|x(t)|^{4} \cdot \frac{\xi}{\mu^{3}}+M \\
& =M-2 \xi|x(t)|^{4},
\end{aligned}
$$

whenever $|x| \geq 1$ and $P(V(t, x(t)))=\mu|x(t)|>|x(s)|=V(s, x(s))$ for $t_{0} \leq s \leq t$.

For $t=\tau_{k}(x(t)), k=1,2, \ldots$ and $|x| \geq 1$,

$$
V\left(t+0, x(t)-\beta_{k} x(t)\right)=\left|\left(1-\beta_{k}\right) x(t)\right| \leq|x(t)|=V(t, x(t)) .
$$

Then all the conditions of Theorem 3.2 are satisfied. Hence the system (4.1) is uniformly ultimately bounded.

\section{References}

[1] A. V. Anokhin, 'On linear impulsive systems for functional differential equations', Soviet Math. Dokl. 33 (1986), 220-223.

[2] D. D. Bainov and A. B. Dishliev, "The phenomenon "beating" of the solutions of impulsive functional differential equations', Commun. Appl. Anal. 1 (1997), 435-441.

[3] D. D. Bainov and P. S. Simeonov, Systems with impulse effect: stability, theory and applications (Ellis Horwood, Chichester, 1989).

[4] D. D. Bainov and P. S. Simeonov, Theory of impulsive differential equations: periodic solutions and applications (Longman, Harlow, 1993).

[5] D. D. Bainov and I. M. Stamova, 'Lypschitz stability of impulsive functional differential equations', ANZIAM J. 42 (2001), 504-515.

[6] D. D. Bainov and I. M. Stamova, 'Global stability of the solutions of impulsive functional differential equations', Kyungpook Math. J. 39 (1999), 239-249.

[7] M.-P. Chen, J. S. Yu and J. H. Shen, 'The persistence of nonoscillatory solutions of delay differential equations under impulsive perturbations', Comput. Math. Appl. 27 (1994), 1-6.

[8] F. Xilin and Z. Liqin, 'On boundedness of solutions of impulsive integro-differential systems with fixed moments of impulse effects', Acta Math. Sci. 17 (1997), 219-229.

[9] J. K. Hale, Theory of functional differential equations (Springer, New York, 1977).

[10] J. K. Hale and V. Lunel, Introduction to functional differential equations (Springer, Berlin, 1993).

[11] V. Lakshmikantham, D. D. Bainov and P. S. Simeonov, Theory of impulsive differential equations (World Scientific, Singapore, 1989).

[12] V. Lakshmikantham, S. Leela and A. A. Martynyuk, Practical stability analysis of nonlinear systems (World Scientific, Singapore, 1990).

[13] Z. Luo and J. Shen, 'Stability and boundedness for impulsive functional differential equations with infinite delays', Nonlinear Anal. 46 (2001), 475-493.

[14] B. S. Razumikhin, Stability of systems with retardation (Nauka, Moscow, 1988) (in Russian).

[15] A. M. Samoilenko and N. A. Perestyuk, Differential equations with impulse effect (Visca Skola, Kiev, 1987) (in Russian).

[16] J. Shen and J. Yan, 'Razumikhin type stability theorems for impulsive functional differential equations', Nonlinear Anal. 33 (1998), 519-531. 
[17] I. M. Stamova and G. T. Stamov, 'Lyapunov-Razumikhin method for impulsive functional differential equations and applications to the population dynamics', J. Comput. Appl. Math. 130 (2001), 163-171.

[18] J. Yan and J. Shen, 'Impulsive stabilization of impulsive functional differential equations by Lyapunov-Razumikhin functions', Nonlinear Anal. 37 (1999), 245-255.

I. M. Stamova, Bourgas Free University, 8000 Burgas, Bulgaria e-mail: stamova@bfu.bg 